\title{
Formulas for Inverse Osculatory Interpolation
}

\author{
Herbert E. Salzer ${ }^{1}$
}

\begin{abstract}
Formulas for inverse osculatory interpolation are obtained by inversion of Hermite's formula. They cover the cases for $n=2(1) 7$, where $n$ is the number of points required in direct osculatory interpolation. The formulas provide an improved means for inverse interpolation in the case where the first derivative is either tabulated alongside the function or is easily obtained.
\end{abstract}

The author has previously given inverse interpolation formulas $[1,2]^{2}$ for finding $x=x_{0}+p h$ from $f(x)$, in terms of $f_{i} \equiv f\left(x_{i}\right)$, where the $x_{i} \equiv x_{0}+i h$ are equally spaced at intervals of $h$. Those formulas were obtained by the inversion of Lagrange's interpolation formula. As the Hermite osculatory interpolation formula $[3,4,5]$ in terms of the function and its first derivative at preassigned points $x_{i}$ is more accurate than the Lagrangian formula, inverse interpolation formulas obtained from the inversion of Hermite's formula would be expected to be more efficient than the corresponding formulas obtained from Lagrange's formula.

There are many tables where the first derivative is either tabulated alongside the function (e. g., Bessel functions of the first or second kind, which give $J_{1}(x)=-J_{0}^{\prime}(x)[6,7]$ or $Y_{1}(x)=-Y_{0}^{\prime}(x)$ [8], respectively, or probability functions [9] ), or where the derivative is very easy to obtain (e. g., tables of the more elementary functions and their integrals, such as tables of sine, cosine, or exponential integrals $[10,11])$. In all such tables, and in many others, the user will find the inverse interpolation formulas given below to be particularly convenient, especially when the tabular interval $h$ is too large for sufficiently accurate inverse interpolation, using the formulas in terms of the functional values alone.

These present formulas give $p$ in terms of $f\left(x_{0}+\right.$ $p h) \equiv f, \quad f\left(x_{i}\right) \equiv f_{i}$, and $f^{\prime}\left(x_{i}\right) \equiv f_{i}^{\prime}$ for $x_{i} \equiv x_{0}+i h$ at equally spaced intervals $h$, where the $i$ ranges from $-[(n-1) / 2]$ to $[n / 2]$, for $n=2(1) 7$, where $n$ is the number of points required in direct osculatory interpolation. Although the direct interpolation formula for $n=6$ and $n=7$ is of the 11th- and 13thdegree accuracy, respectively, the inversion formula for $p$ that is given below does not go beyond the 10th- degree terms (and in most practical problems it is very rarely that one will go that far; in fact, the first few terms will usually suffice). Thus we do not use the coefficient of $p^{11}$ in the 6 -point Hermite formula nor the coefficients of $p^{11}, p^{12}$, and $p^{13}$ in the 7-point Hermite formula. For every $n$, we define $r=\left(f-f_{0}\right) / h f_{0}^{\prime}$, and corresponding to $n=2(1) 7$, quantities $s, t, u, v, w, x, y, z$, and $\bar{z}$ are defined as follows:

$$
\begin{gathered}
n=2 \\
s=\left(-3\left\{f_{0}-f_{1}\right\}-h\left\{2 f_{0}^{\prime}+f_{1}^{\prime}\right\}\right) / h f_{0}^{\prime}, \\
t=\left(2\left\{f_{0}-f_{1}\right\}+h\left\{f_{0}^{\prime}+f_{1}^{\prime}\right\}\right) / h f_{0}^{\prime}, \\
u=v=w=x=y=z=\bar{z}=0 . \\
n=3 \\
s=\left(f_{-1}-2 f_{0}+f_{1}+\frac{h}{4}\left\{f_{-1}^{\prime}-f_{1}^{\prime}\right\}\right) / h f_{0}^{\prime}, \\
t=\left(-\frac{5}{4}\left\{f_{-1}-f_{1}\right\}-\frac{h}{4}\left\{f_{-1}^{\prime}+8 f_{0}^{\prime}+f_{1}^{\prime}\right\}\right) / h f_{0}^{\prime}, \\
u=\left(-\frac{1}{2}\left\{f_{-1}-2 f_{0}+f_{1}\right\}-\frac{h}{4}\left\{f_{-1}^{\prime}-f_{1}^{\prime}\right\}\right) / h f_{0}^{\prime}, \\
v=\left(\frac{3}{4}\left\{f_{-1}-f_{1}\right\}+\frac{h}{4}\left\{f_{-1}^{\prime}+4 f_{0}^{\prime}+f_{1}^{\prime}\right\}\right) / h f_{0}^{\prime}, \\
w=x=y=z=\bar{z}=0 .
\end{gathered}
$$

${ }_{1}$ Present address, Diamond Ordnance FuzelLaboratory, Department of 


$$
\begin{aligned}
& s=\left(\frac{1}{108}\left\{56 f_{-1}-297 f_{0}+216 f_{1}+25 f_{2}\right\}+\frac{h}{18}\left\{2 f_{-1}^{\prime}-18 f_{0}^{\prime}-18 f_{1}^{\prime}-f_{2}^{\prime}\right\}\right) / h f_{0}^{\prime}, \\
& t=\left(-\frac{1}{108}\left\{124 f_{-1}-27 f_{0}-108 f_{1}+11 f_{2}\right\}-\frac{h}{36}\left\{8 f_{-1}^{\prime}+63 f_{0}^{\prime}-f_{2}^{\prime}\right\}\right) / h f_{0}^{\prime}, \\
& u=\left(\frac{1}{54}\left\{25\left(f_{-1}-f_{2}\right)+135\left(f_{0}-f_{1}\right)\right\}+\frac{h}{36}\left\{f_{-1}^{\prime}+72 f_{0}^{\prime}+63 f_{1}^{\prime}+4 f_{2}^{\prime}\right\}\right) / h f_{0}^{\prime}, \\
& v=\left(\frac{1}{108}\left\{59 f_{-1}-54 f_{0}-27 f_{1}+22 f_{2}\right\}+\frac{h}{36}\left\{7 f_{-1}^{\prime}+18 f_{0}^{\prime}-9 f_{1}^{\prime}-2 f_{2}^{\prime}\right\}\right) / h f_{0}^{\prime}, \\
& w=\left(-\frac{1}{108}\left\{52 f_{-1}+81 f_{0}-108 f_{1}-25 f_{2}\right\}-\frac{h}{36}\left\{5 f_{-1}^{\prime}+36 f_{0}^{\prime}+27 f_{1}^{\prime}+2 f_{2}^{\prime}\right\}\right) / h f_{0}^{\prime}, \\
& x=\left(\frac{1}{108}\left\{11\left(f_{-1}-f_{2}\right)+27\left(f_{0}-f_{1}\right)\right\}+\frac{h}{36}\left\{f_{-1}^{\prime}+9 f_{0}^{\prime}+9 f_{1}^{\prime}+f_{2}^{\prime}\right\}\right) / h f_{0}^{\prime}, \\
& y=z=\bar{z}=0 .
\end{aligned}
$$

$$
n=5
$$

$$
\begin{aligned}
& s=\left(\frac{1}{108}\left\{7\left(f_{-2}+f_{2}\right)+128\left(f_{-1}+f_{1}\right)-270 f_{0}\right\}+\frac{h}{72}\left\{\left(f_{-2}^{\prime}-f_{2}^{\prime}\right)+32\left(f_{-1}^{\prime}-f_{1}^{\prime}\right)\right\}\right) / h f_{0}^{\prime}, \\
& t=\left(-\frac{1}{864}\left\{31\left(f_{-2}-f_{2}\right)+1408\left(f_{-1}-f_{1}\right)\right\}-\frac{h}{144}\left\{\left(f_{-2}^{\prime}+f_{2}^{\prime}\right)+64\left(f_{-1}^{\prime}+f_{1}^{\prime}\right)+360 f_{0}^{\prime}\right\}\right) / h f_{0}^{\prime}, \\
& u=\left(-\frac{1}{288}\left\{41\left(f_{-2}+f_{2}\right)+256\left(f_{-1}+f_{1}\right)-594 f_{0}\right\}-\frac{h}{96}\left\{3\left(f_{-2}^{\prime}-f_{2}^{\prime}\right)+64\left(f_{-1}^{\prime}-f_{1}^{\prime}\right)\right\}\right) / h f_{0}^{\prime},
\end{aligned}
$$$$
v=\left(\frac{1}{1152}\left\{91\left(f_{-2}-f_{2}\right)+1792\left(f_{-1}-f_{1}\right)\right\}+\frac{h}{192}\left\{3\left(f_{-2}^{\prime}+f_{2}^{\prime}\right)+128\left(f_{-1}^{\prime}+f_{1}^{\prime}\right)+396 f_{0}^{\prime}\right\}\right) / h f_{0}^{\prime},
$$$$
w=\left(\frac{1}{144}\left\{13\left(f_{-2}+f_{2}\right)+32\left(f_{-1}+f_{1}\right)-90 f_{0}\right\}+\frac{h}{48}\left\{\left(f_{-2}^{\prime}-f_{2}^{\prime}\right)+12\left(f_{-1}^{\prime}-f_{1}^{\prime}\right)\right\}\right) / h f_{0}^{\prime},
$$$$
x=\left(-\frac{1}{576}\left\{29\left(f_{-2}-f_{2}\right)+272\left(f_{-1}-f_{1}\right)\right\}-\frac{h}{96}\left\{\left(f_{-2}^{\prime}+f_{2}^{\prime}\right)+24\left(f_{-1}^{\prime}+f_{1}^{\prime}\right)+60 f_{0}^{\prime}\right\}\right) / h f_{0}^{\prime},
$$$$
y=\left(-\frac{1}{864}\left\{11\left(f_{-2}+f_{2}\right)+16\left(f_{-1}+f_{1}\right)-54 f_{0}\right\}-\frac{h}{288}\left\{\left(f_{-2}^{\prime}-f_{2}^{\prime}\right)+8\left(f_{-1}^{\prime}-f_{1}^{\prime}\right)\right\}\right) / h f_{0}^{\prime},
$$$$
z=\left(\frac{1}{3456}\left\{25\left(f_{-2}-f_{2}\right)+160\left(f_{-1}-f_{1}\right)\right\}+\frac{h}{576}\left\{\left(f_{-2}^{\prime}+f_{2}^{\prime}\right)+16\left(f_{-1}^{\prime}+f_{1}^{\prime}\right)+36 f_{0}^{\prime}\right\}\right) / h f_{0}^{\prime},
$$

$\bar{z}=0$.

$$
\begin{gathered}
n=6 \\
s=\left(\frac{1}{3000}\left\{76 f_{-2}+2375 f_{-1}-8500 f_{0}+5000 f_{1}+1000 f_{2}+49 f_{3}\right\}\right. \\
\left.+\frac{h}{600}\left\{3 f_{-2}^{\prime}+150 f_{-1}^{\prime}-400 f_{0}^{\prime}-600 f_{1}^{\prime}-75 f_{2}^{\prime}-2 f_{3}^{\prime}\right\}\right) / h f_{0}^{\prime}, \\
t=\left(-\frac{1}{108000}\left\{3327 f_{-2}+169500 f_{-1}-8000 f_{0}-168000 f_{1}+2625 f_{2}+548 f_{3}\right\}\right. \\
\left.-\frac{h}{3600}\left\{21 f_{-2}^{\prime}+1500 f_{-1}^{\prime}+8600 f_{0}^{\prime}+1200 f_{1}^{\prime}-75 f_{2}^{\prime}-4 f_{3}^{\prime}\right\}\right) / h f_{0}^{\prime},
\end{gathered}
$$




$$
\begin{aligned}
& u=\left(-\frac{1}{21600}\left\{943 f_{-2}-2050 f_{-1}-62550 f_{0}+45200 f_{1}+17575 f_{2}+882 f_{3}\right\}\right. \\
& \left.-\frac{h}{1440}\left\{13 f_{-2}^{\prime}+260 f_{-1}^{\prime}-2400 f_{0}^{\prime}-2960 f_{1}^{\prime}-445 f_{2}^{\prime}-12 f_{3}^{\prime}\right\}\right) / h f_{0}^{\prime}, \\
& v=\left(\frac{1}{86400}\left\{5729 f_{-2}+121400 f_{-1}-16000 f_{0}-118400 f_{1}-6175 f_{2}+1096 f_{3}\right\}\right. \\
& \left.+\frac{h}{2880}\left\{37 f_{-2}^{\prime}+1720 f_{-1}^{\prime}+5140 f_{0}^{\prime}+1120 f_{1}^{\prime}-155 f_{2}^{\prime}-8 f_{3}^{\prime}\right\}\right) / h f_{0}^{\prime}, \\
& w=\left(\frac{1}{144000}\left\{1274 f_{-2^{--}} 84875 f_{-1}-189000 f_{0}+175000 f_{1}+92750 f_{2}+4851 f_{3}\right\}\right. \\
& \left.+\frac{h}{14400}\left\{36 f_{-2}^{\prime}-2175 f_{-1}^{\prime}-19800 f_{0}^{\prime}-20100 f_{1}^{\prime}-3600 f_{2}^{\prime}-99 f_{3}^{\prime}\right\}\right) / h f_{0}^{\prime}, \\
& x=\left(-\frac{1}{144000}\left\{5743 f_{-2}+50125 f_{-1}-22000 f_{0}-46000 f_{1}+10625 f_{2}+1507 f_{3}\right\}\right. \\
& \left.-\frac{h}{4800}\left\{39 f_{-2}^{\prime}+925 f_{-1}^{\prime}+1900 f_{0}^{\prime}+100 f_{1}^{\prime}-225 f_{2}^{\prime}-11 f_{3}^{\prime}\right\}\right) / h f_{0}^{\prime},
\end{aligned}
$$$$
y=\left(\frac{1}{14400}\left\{172 f_{-2}+3275 f_{-1}+3900 f_{0}-4600 f_{1}-2600 f_{2}-147 f_{3}\right\}\right.
$$$$
\left.+\frac{h}{480}\left\{f_{-2}^{\prime}+45 f_{-1}^{\prime}+200 f_{0}^{\prime}+180 f_{1}^{\prime}+35 f_{2}^{\prime}+f_{3}^{\prime}\right\}\right)\left(h f_{0}^{\prime},\right.
$$$$
z=\left(\frac{1}{86400}\left\{351 f_{-2}+750 f_{-1}-4000 f_{0}+2625 f_{2}+274 f_{3}\right\}\right.
$$$$
\left.+\frac{h}{2880}\left\{3 f_{-2}^{\prime}+30 f_{-1}^{\prime}-20 f_{0}^{\prime}-120 f_{1}^{\prime}-45 f_{2}^{\prime}-2 f_{3}^{\prime}\right\}\right) / h f_{0}^{\prime},
$$$$
\bar{z}=\left(-\frac{1}{432000}\left\{1066 f_{-2}+10625 f_{-1}+9000 f_{0}-13000 f_{1}-7250 f_{2}-441 f_{3}\right\}\right.
$$$$
\left.-\frac{h}{14400}\left\{8 f_{-2}^{\prime}+175 f_{-1}^{\prime}+600 f_{0}^{\prime}+500 f_{1}^{\prime}+100 f_{2}^{\prime}+3 f_{3}^{\prime}\right\}\right) / h f_{0}^{\prime} .
$$$$
n=7
$$$$
s=\left(\frac{1}{36000}\left\{157\left(f_{-3}+f_{3}\right)+4968\left(f_{-2}+f_{2}\right)+43875\left(f_{-1}+f_{1}\right)-98000 f_{0}\right\}\right.
$$$$
\left.+\frac{h}{1200}\left\{\left(f_{-3}^{\prime}-f_{3}^{\prime}\right)+54\left(f_{-2}^{\prime}-f_{2}^{\prime}\right)+675\left(f_{-1}^{\prime}-f_{1}^{\prime}\right)\right\}\right) / h f_{0}^{\prime},
$$$$
t=\left(-\frac{1}{108000}\left\{167\left(f_{-3}-f_{3}\right)+8667\left(f_{-2}-f_{2}\right)+192375\left(f_{-1}-f_{1}\right)\right\}\right.
$$$$
\left.-\frac{h}{3600}\left\{\left(f_{-3}^{\prime}+f_{3}^{\prime}\right)+81\left(f_{-2}^{\prime}+f_{2}^{\prime}\right)+2025\left(f_{-1}^{\prime}+f_{1}^{\prime}\right)+9800 f_{0}^{\prime}\right\}\right) / h f_{0}^{\prime},
$$

$u=\left(-\frac{1}{648000}\left\{7339\left(f_{-3}+f_{3}\right)+213786\left(f_{-2}+f_{2}\right)+631125\left(f_{-1}+f_{1}\right)-1704500 f_{0}\right\}\right.$

$$
\left.-\frac{h}{21600}\left\{47\left(f_{-3}^{\prime}-f_{3}^{\prime}\right)+2403\left(f_{-2}^{\prime}-f_{2}^{\prime}\right)+20925\left(f_{-1}^{\prime}-f_{1}^{\prime}\right)\right\}\right) / h f_{0}^{\prime},
$$

$$
\begin{aligned}
v=\left(\frac{1}{1296000}\left\{5206\left(f_{-3}-f_{3}\right)+249831\left(f_{-2}-f_{2}\right)+2517750\left(f_{-1}-f_{1}\right)\right\}\right. \\
\left.+\frac{h}{129600}\left\{94\left(f_{-3}^{\prime}+f_{3}^{\prime}\right)+7209\left(f_{-2}^{\prime}+f_{2}^{\prime}\right)+125550\left(f_{-1}^{\prime}+f_{1}^{\prime}\right)+340900 f_{0}^{\prime}\right\}\right) / h f_{0}^{\prime},
\end{aligned}
$$

$w=\left(\frac{1}{5184000}\left\{52109\left(f_{-3}+f_{3}\right)+1310016\left(f_{-2}+f_{2}\right)+1525875\left(f_{-1}+f_{1}\right)-5776000 f_{0}\right\}\right.$

$$
\left.+\frac{h}{172800}\left\{337\left(f_{-3}^{\prime}-f_{3}^{\prime}\right)+15648\left(f_{-2}^{\prime}-f_{2}^{\prime}\right)+88275\left(f_{-1}^{\prime}-f_{1}^{\prime}\right)\right\}\right) / h f_{0}^{\prime},
$$




$$
\begin{aligned}
x=\left(-\frac{1}{5184000}\{\right. & \left.18493\left(f_{-3}-f_{3}\right)+772368\left(f_{-2}-f_{2}\right)+4174125\left(f_{-1}-f_{1}\right)\right\} \\
& \left.-\frac{h}{518400}\left\{337\left(f_{-3}^{\prime}+f_{3}^{\prime}\right)+23472\left(f_{-2}^{\prime}+f_{2}^{\prime}\right)+264825\left(f_{-1}^{\prime}+f_{1}^{\prime}\right)+577600 f_{0}^{\prime}\right\}\right) / h f_{0}^{\prime},
\end{aligned}
$$

$$
\begin{aligned}
y=\left(-\frac{1}{1728000}\left\{6217\left(f_{-3}+f_{3}\right)+\right.\right. & \left.117408\left(f_{-2}+f_{2}\right)+72375\left(f_{-1}+f_{1}\right)-392000 f_{0}\right\} \\
& \left.-\frac{h}{57600}\left\{41\left(f_{-3}^{\prime}-f_{3}^{\prime}\right)+1584\left(f_{-2}^{\prime}-f_{2}^{\prime}\right)+6675\left(f_{-1}^{\prime}-f_{1}^{\prime}\right)\right\}\right) / h f_{0}^{\prime},
\end{aligned}
$$

$$
\begin{gathered}
z=\left(\frac{1}{1728000}\left\{2209\left(f_{-3}-f_{3}\right)+70584\left(f_{-2}-f_{2}\right)+272625\left(f_{-1}-f_{1}\right)\right\}\right. \\
\left.+\frac{h}{172800}\left\{41\left(f_{-3}^{\prime}+f_{3}^{\prime}\right)+2376\left(f_{-2}^{\prime}+f_{2}^{\prime}\right)+20025\left(f_{-1}^{\prime}+f_{1}^{\prime}\right)+39200 f_{0}^{\prime}\right\}\right) / h f_{0}^{\prime} \\
\bar{z}=\left(\frac{1}{5184000}\left\{2783\left(f_{-3}+f_{3}\right)+38592\left(f_{-2}+f_{2}\right)+14625\left(f_{-1}+f_{1}\right)-112000 f_{0}\right\}\right.
\end{gathered}
$$$$
\left.+\frac{h}{172800}\left\{19\left(f_{-3}^{\prime}-f_{3}^{\prime}\right)+576\left(f_{-2}^{\prime}-f_{2}^{\prime}\right)+2025\left(f_{-1}^{\prime}-f_{1}^{\prime}\right)\right\}\right) / h f_{0}^{\prime} .
$$

For every $n$, the formula for $p$ is the following:

$$
\begin{aligned}
p= & -r^{2} s+r^{3}\left(2 s^{2}-t\right)+r^{4}\left(-5 s^{3}+5 s t-u\right)+r^{5}\left(14 s^{4}-21 s^{2} t+3 t^{2}+6 s u-v\right)+r^{6}\left(-42 s^{5}+84 s^{3} t\right. \\
& \left.-28 s t^{2}-28 s^{2} u+7 t u+7 s v-w\right)+r^{7}\left(132 s^{6}-330 s^{4} t+180 s^{2} t^{2}+120 s^{3} u-12 t^{3}-72 s t u-36 s^{2} v+4 u^{2}\right. \\
& +8 t v+8 s w-x)+r^{8}\left(-429 s^{7}+1287 s^{5} t-990 s^{3} t^{2}-495 s^{4} u+495 s^{2} t u+165 s t^{3}+165 s^{3} v-45 t^{2} u\right. \\
& \left.-45 s u^{2}-90 s t v-45 s^{2} w+9 u v+9 t w+9 s x-y\right)+r^{9}\left(1430 s^{8}-5005 s^{6} t+5005 s^{4} t^{2}+2002 s^{5} u\right. \\
& -1430 s^{2} t^{3}-2860 s^{3} t u-715 s^{4} v+55 t^{4}+660 s t^{2} u+330 s^{2} u^{2}+660 s^{2} t v+220 s^{3} w-55 t u^{2}-55 t^{2} v \\
& \left.-110 s u v-110 s t w-55 s^{2} x+5 v^{2}+10 u w+10 t x+10 s y-z\right)+r^{10}\left(-4862 s^{9}+19448 s^{7} t-24024 s^{5} t^{2}\right. \\
& -8008 s^{6} u+10010 s^{3} t^{3}+15015 s^{4} t u+3003 s^{5} v-1001 s t^{4}-6006 s^{2} t^{2} u-2002 s^{3} u^{2}-4004 s^{3} t v \\
& -1001 s^{4} w+286 t^{3} u+858 s t u^{2}+858 s^{2} t w+858 s t^{2} v+858 s^{2} u v+286 s^{3} x-22 u^{3}-132 t u v-66 t^{2} w \\
& \left.-66 s v^{2}-132 s u w-132 s t x-66 s^{2} y+11 v w+11 u x+11 t y+11 s z-\bar{z}\right)+\cdots
\end{aligned}
$$

[1] H. E. Salzer, A new formula for inverse interpolation, Bul. Am. Math. Soc. 50, 513 (1944).

[2] H. E. Salzer, Inverse interpolation for eight-, nine-, ten-, and eleven-point direct interpolation, J. Math. Phys. 24, 106 (1945).

[3] T. Fort, Finite differences, p. 85 (Clarendon Press, Oxford, England, 1948).

[4] J. F. Steffensen, Interpolation, p. 33 (Williams \& Wilkins, Baltimore, Md., 1927).

[5] H. E. Salzer, New formulas for facilitating osculatory interpolation, J. Research NBS 52, 211 (1954) RP2491.

[6] National Bureau of Standards, Table of the Bessel functions $J_{0}(z)$ and $J_{1}(z)$ for complex arguments (Columbia University Press, New York, N. Y., 1943;2d ed. 1947).

[7] Harvard Computation Laboratory, The annals of the computation laboratory of Harvard University III, Tables of the Bessel functions of the first kind of orders zero and one (Harvard University Press, Cambridge, Mass., 1947).
[8] National Bureau of Standards, Table of the Bessel functions $Y_{0}(z)$ and $Y_{1}(z)$ for complex arguments (Columbia University Press, New York, N. Y., 1950).

[9] Tables of normal probability functions, NBS AMS23 (1953); Tables of the error function and its derivative, NBS AMS41 (1954).

[10] Tables of sine, cosine, and exponential integrals, NBS MT5; NBS MT6 (1940).

[11] Table of sine and cosine integrals for arguments from 10 to 100 , NBS AMS32 (1954).

Washington, September 27, 1955. 